\title{
Rh(II)-Catalyzed Enantioselective Cyclopropanation of Olefins with Dimethyl malonate via in situ Generated Phenyliodonium Ylide
}

\author{
Paul Müller* and Ashraf Ghanem \\ Department of Organic Chemistry, University of Geneva, 30, Quai Ernest-Ansermet \\ CH-1211 Geneva 4, Switzerland
}

Paul.muller@chiorg.unige.ch

\section{Supporting Material}

\section{Synthesis of iodosyl benzene, $\mathrm{PhI}=\mathrm{O}^{1}$}

To finely powdered diacetoxyiodobenzene $(32.2 \mathrm{~g}, 0.10 \mathrm{~mol})$ was added $3 \mathrm{~N} \mathrm{NaOH}$ $(150 \mathrm{~mL})$ in $5 \mathrm{~min}$. with vigorous stirring, and the lumps of solid, which formed where macerated with a spatula. The reaction mixture was stirred for $45 \mathrm{~min}$ and then diluted with $\mathrm{H}_{2} \mathrm{O}(100 \mathrm{~mL})$. The crude yellow solid was collected by filtration, washed with $\mathrm{H}_{2} \mathrm{O}(3 \times 100 \mathrm{~mL})$ and dried under vacuum. It was suspended in $\mathrm{CHCl}_{3}$ $(75 \mathrm{~mL})$, macerated and separated by filtration. The crude $\mathrm{PhI}=\mathrm{O}$ was air-dried and used without further purification.

\section{General procedure for the asymmetric cyclopropanation of olefins}

Dimethyl malonate $(0.01 \mathrm{~mol})$ is added to a mixture of iodosyl benzene (1.4 equivalent), olefins (10 equivalent), $\mathrm{MgO}$ (2.3 equivalent), rhodium (II) catalyst (5 mol\%) and $250 \mathrm{mg}$ molecular sieves $4 \AA$ in dichloromethane $(10 \mathrm{ml})$. The reaction mixture is stirred under argon for $24 \mathrm{hrs.} 100 \mu \mathrm{l}$ samples were taken after several time intervals. The samples were filtered using a syringe filter holder $(0.2 \mu \mathrm{m}$ pore size $)$ and the organic layer was diluted with $100 \mu \mathrm{l}$ of dichloromethane and analysed by GC. The reaction progress was monitored qualitatively by thin layer chromatography using heptane/ethyl acetate $(5: 1 \mathrm{v} / \mathrm{v})$ as eluent. An aliquot of the supernatant was used for GC. When maximum conversion was reached, the reaction was terminated by filtration through celite. The residue on the celite was washed twice with dichloromethane. Evaporation of the combined filtrates under reduced pressure 
followed by chromatography on silica gel column with heptane/ethyl acetate $(5: 1 \mathrm{v} / \mathrm{v})$ as eluent afforded the desired cyclopropane derivatives.

\section{Enantioselective gas-chromatographic analysis}

A gas chromatograph (Hewlett Packard 580) equipped with a flame ionization detector (FID) was used in the determination of the enantiomeric excess of the enantiomerically enriched cyclopropane derivatives. . The chiral stationary phase was either (heptakis-(2,3-di-O-methyl-6-O-tert-butyldimethylsilyl)- $\beta$-cyclodextrin, $20 \%$ (w/w)), which was dissolved in PS 86 (Gelest, ABCR GmbH \& Co., Karlsruhe, Germany) and coated on a 25 or $10 \mathrm{~m}$ x $0.25 \mathrm{~mm}$ fused silica capillary column $(0.25$ $\mu \mathrm{m}$ film thickness) according to literature procedure, ${ }^{2}$ or commercially available chirasil- $\beta$-dex with a new 11-spacer (C11-Chirasil-Dex) coated on a non-deactivated $19 \mathrm{~m}$ x $0.25 \mathrm{~mm}$ fused silica capillary column $(0.25 \mu \mathrm{m}$ film thickness $){ }^{3}$ Analytical conditions were: injector temperature, $200{ }^{\circ} \mathrm{C}$; FID temperature, $250^{\circ} \mathrm{C}$; oven temperature variable depending uèpon the sample. Hydrogen was used as the carrier gas (40 KPa column head pressure). Temperature program: $80{ }^{\circ} \mathrm{C}$ for $30 \mathrm{~min}$., then 1 ${ }^{\circ} \mathrm{C} / \mathrm{min}$. until $180{ }^{\circ} \mathrm{C}$. The products were identified by comparaison with those prepared using either $\left[\mathrm{Rh}_{2}(\mathrm{OAc})_{4}\right]$ or $\left[\mathrm{Cu}_{2}(\mathrm{acac})_{4}\right]$ catalysts.

\section{Characterization of cyclopropanes}

4.1. $(R)$-Dimethyl 2-phenylcyclopropane-1,1-dicarboxylate $(3 a){ }^{4}[\alpha]_{\mathrm{D}}{ }^{24}=+57(c$ $0.3, \mathrm{PhH}$ for $82 \%$ ee $)$; Lit. $^{5}[\alpha]_{\mathrm{D}}{ }^{23}=-124(\mathrm{c}=2.23, \mathrm{PhH}$ for $(S)-3 \mathrm{a}) .{ }^{1} \mathrm{H}$ NMR $(400$ $\left.\mathrm{MHZ}, \mathrm{CDCl}_{3}\right): 1.71(d d, J=4, J=41 \mathrm{H}) ; 2.18(d d, J=4, J=41 \mathrm{H}) ; 3.21(t, J=8,1 \mathrm{H})$; $3.34(s, 3 \mathrm{H}) ; 3.76(s, 3 \mathrm{H}) ; 7.19-7.28(m, 5 \mathrm{H}) .{ }^{13} \mathrm{C} \mathrm{NMR}\left(100 \mathrm{MHZ}, \mathrm{CDCl}_{3}\right): 19.1(t)$; $32.6(d) ; 37.2(s) ; 52.2(q) ; 52.6(q) ; 127.4(d) ; 128.2(d) ; 128.4(d) ; 134.5(s) ; 164.8$ $(s) ;$ 169.4(s). HR MS: $234.0897\left(\mathrm{C}_{13} \mathrm{H}_{14} \mathrm{O}_{4}{ }^{+}\right.$; calc. 234.0892). Enantiomer separation: chirasil- $\beta$-dex $(30 \mathrm{~m})$ column, oven temperature: $80{ }^{\circ} \mathrm{C}$ for $30 \mathrm{~min}$, then $1{ }^{\circ} \mathrm{C} / \mathrm{min}$ until $180{ }^{\circ} \mathrm{C} . \mathrm{t}_{\mathrm{R}}[(R)-\mathbf{3 a}]=54.3 \mathrm{~min}, \mathrm{t}_{\mathrm{R}}[(S)-\mathbf{3 a}]=54.7 \mathrm{~min}$.

\section{2. (R)-Dimethyl 2-(4-methylphenyl)-cyclopropane-1,1-dicarboxylate (3b). ${ }^{4}$} $[\alpha]_{\mathrm{D}}{ }^{24}=+62(c 0.2, \mathrm{PhH}$ for $87 \%$ ee $) ;{ }^{1} \mathrm{H}$ NMR: $\left(400 \mathrm{MHZ}, \mathrm{CDCl}_{3}\right): 1.79(d d, J=4$, $J=41 \mathrm{H}), 2.18(d d, J=8, J=81 \mathrm{H}) ; 2.20(s, 3 \mathrm{H}) ; 3.21(t, J=12,1 \mathrm{H}) ; 3.42(s, 3 \mathrm{H}) ; 3.81$ 
$(s, 3 \mathrm{H}) ; 7.15(s, 4 \mathrm{H}) .{ }^{13} \mathrm{C}$ NMR (100 MHZ, $\left.\mathrm{CDCl}_{3}\right): 19.0(t) ; 20.9(q) ; 32.3(d) ; 37.0$ $(s) ; 52.0(q) ; 52.6(q) ; 128.1(d) ; 128.7(d) ; 131.3(s) ; 136.9(s) ; 166.9(s) ; 170.1(s)$. HR MS: 248.10530 $\left(\mathrm{C}_{14} \mathrm{H}_{16} \mathrm{O}_{4}{ }^{+}\right.$; calc. 248.10491). Anal. calc. for $\mathrm{C}_{14} \mathrm{H}_{16} \mathrm{O}_{4}(248.28)$ : C 67.73, H 6.50; found: C 67.50, H 6.58. Enantiomer separation: chirasil- $\beta$-dex (30 m) column, oven temperature: $100{ }^{\circ} \mathrm{C}$ for $120 \mathrm{~min}$, then $0.5{ }^{\circ} \mathrm{C} / \mathrm{min}$ until $180{ }^{\circ} \mathrm{C} \cdot \mathrm{t}_{\mathrm{R}}$ $[(R)-3 \mathbf{b}]=209.4 \mathrm{~min}, \mathrm{t}_{\mathrm{R}}[(S)-3 \mathbf{b}]=211.1 \mathrm{~min}$. HPLC: CIRACEL-OD column, with $n$-hexane/isopropanol 90:10. $\mathrm{t}_{\mathrm{R}}[(S)-3 \mathbf{b}]=24.5 \mathrm{~min}, \mathrm{t}_{\mathrm{R}}[(R)-3 \mathrm{~b}]=25.5 \mathrm{~min}$.

\section{3. (R)-Dimethyl 2-(4-chlorophenyl)-cyclopropane-1,1-dicarboxylate $(3 \mathrm{c}) .^{6}[\alpha]_{\mathrm{D}}{ }^{24}$} $=+46.1\left(c 0.7, \mathrm{PhH}\right.$ for $65 \%$ ee); Lit. $^{7}[\alpha]_{\mathrm{D}}=117.0$ (for $(R)-3 \mathrm{c}$ of $100 \%$ ee). ${ }^{1} \mathrm{H}$ NMR (400 MHZ, $\left.\mathrm{CDCl}_{3}\right): 1.72(d d, J=4, J=41 \mathrm{H}) ; 2.13(d d, J=8,1 \mathrm{H}) ; 3.16(t, J=8$, $1 \mathrm{H}) ; 3.40(s, 3 \mathrm{H}) ; 3.79(s, 3 \mathrm{H}) ; 7.12(d, J=8,2 \mathrm{H}) ; 7.23(d, J=8,2 \mathrm{H}) .{ }^{13} \mathrm{C}$ NMR $(100$ MHZ, $\left.\mathrm{CDCl}_{3}\right) ; 18.1(t) ; 30.7(d) ; 36.2(s) ; 51.9(q) ; 52.4(q) ; 127.6(d) ; 129.0(d)$; $132.1(s), 132.3(s) ; 165.8(s) ; 168.9(s)$. HR MS: $268.0492\left(\mathrm{C}_{13} \mathrm{H}_{13} \mathrm{O}_{4}{ }^{35} \mathrm{Cl}^{+}\right.$; calc. 268.0502). Anal. calc. for $\mathrm{C}_{13} \mathrm{H}_{13} \mathrm{ClO}_{4}$ (268.70): C 58.11, $\mathrm{H} 4.88$; found: $\mathrm{C} 58.0, \mathrm{H}$ 4.70. Enantiomer separation: chirasil- $\beta$-dex $(30 \mathrm{~m})$ column, oven temperature: $80{ }^{\circ} \mathrm{C}$ for $30 \mathrm{~min}$, then $1{ }^{\circ} \mathrm{C} / \mathrm{min}$ until $180{ }^{\circ} \mathrm{C} . \mathrm{t}_{\mathrm{R}}[(R)-3 \mathrm{c}]=113.7 \mathrm{~min}, \mathrm{t}_{\mathrm{R}}[(S)-\mathbf{3 c}]=114.9$ min.

4.4. (R)-Dimethyl 2-(4-bromophenyl)-cyclopropane-1,1-dicarboxylate (3d). $[\alpha]_{\mathrm{D}}{ }^{24}$ $=+57(c 0.2, \mathrm{PhH}$ for $90 \%$ ee $),{ }^{1} \mathrm{H}$ NMR $\left(400 \mathrm{MHZ}, \mathrm{CDCl}_{3}\right): 1.75(d d, J=4, J=4$ 1H): $2.15(d d, J=8, J=81 \mathrm{H}) ; 3.21(t, J=8,1 \mathrm{H}) ; 3.42(s, 3 \mathrm{H}) ; 3.81(s, 3 \mathrm{H}) ; 7.08(d$, $J=4,2 \mathrm{H}) ; 7.40\left(d, J=8\right.$ 2H), ${ }^{13} \mathrm{C}$ NMR (100 MHZ, $\left.\mathrm{CDCl}_{3}\right): 19.1(t) ; 32.6(d) ; 37.2$ $(s) ; 52.2(q) ; 52.6(q) ; 120.4(s) ; 129.1(d) ; 129.9(d) ; 132.6(s) ; 165.8(s) ; 168.9(s)$.

MS: 314(M+14), 312 (M+14), 282 (82), 280 (28), 250 (20), 148 (19), 202 (19), 201 (100), 199 (69), 195 (26), 193 (26), 173 (20), 170 (27), 145 (27), 129 (13), 115 (62), 114 (23), 113 (10), 103 (12), 102 (10), 89 (16), 77 (14), 63 (17), 59 (45), 51 (10). HR MS: $312.0010\left(\mathrm{C}_{13} \mathrm{H}_{13} \mathrm{O}_{4}{ }^{79} \mathrm{Br}^{+}\right.$; calc. 311.9997$) ; 313.9998\left(\mathrm{C}_{13} \mathrm{H}_{13} \mathrm{O}_{4}{ }^{81} \mathrm{Br}^{+}\right.$; calc. 313.9977). Anal. calc. for $\mathrm{C}_{13} \mathrm{H}_{13} \mathrm{BrO} 4$ (311.9): $\mathrm{C} 50.86, \mathrm{H} 4.16$; found: $\mathrm{C} 50.64, \mathrm{H}$ 4.10. Enantiomer separation: chirasil- $\beta$-dex $(30 \mathrm{~m})$ column, oven temperature: $80{ }^{\circ} \mathrm{C}$ for $30 \mathrm{~min}$, then $1{ }^{\circ} \mathrm{C} / \mathrm{min}$ until $180{ }^{\circ} \mathrm{C}$. $\mathrm{t}_{\mathrm{R}}[(R)-\mathbf{3 d})=127.2 \mathrm{~min}, \mathrm{t}_{\mathrm{R}}=[(S)-\mathbf{3 d}) 128.2$ min. 


\section{5. (R)-Dimethyl 2-(4-trifluoromethylphenyl)-cyclopropane-1,1-dicarboxylate}

(3e). ${ }^{8}[\alpha]_{\mathrm{D}}{ }^{24}=+42\left(c 0.7, \mathrm{PhH}\right.$ for $87 \%$ ee). ${ }^{1} \mathrm{H}$ NMR (400 MHZ, $\left.\mathrm{CDCl}_{3}\right): 1.77(d d$, $\mathrm{J}=4, \mathrm{~J}=41 \mathrm{H}) ; 2.18(d d, J=8, J=81 \mathrm{H}) ; 3.24(t, J=8,1 \mathrm{H}) ; 3.40(s, 3 \mathrm{H}) ; 3.81(s, 3 \mathrm{H})$; $7.38(d, J=8,2 \mathrm{H}) ; 7.50(d, J=82 \mathrm{H}) .{ }^{13} \mathrm{C} \mathrm{NMR}\left(100 \mathrm{MHZ}, \mathrm{CDCl}_{3}\right): 19.1(t) ; 29.6(s)$; $31.6(d) ; 37.3(s) ; 52.2(q) ; 52.6(q) ; 124.1(s) ; 125.4(s) ; 128.1(d) ; 128.9(s) ; 134.6$ $(d) ; 165.8(s) ; 168.9(s)$. Enantiomer separation: chirasil- $\beta$-dex $(30 \mathrm{~m})$ column, oven temperature: $80{ }^{\circ} \mathrm{C}$ for $30 \mathrm{~min}$, then $1{ }^{\circ} \mathrm{C} / \mathrm{min}$ until $180{ }^{\circ} \mathrm{C} . \mathrm{t}_{\mathrm{R}}[(R)-3 \mathrm{e}]=112.5 \mathrm{~min}, \mathrm{t}_{\mathrm{R}}$ $[(S)-3 \mathbf{e})]=113.1 \mathrm{~min}$.

4.6. (S)-Dimethyl 2-propylcyclopropane-1,1-dicarboxylate $(3 \mathrm{f}) \cdot{ }^{9}[\alpha]_{\mathrm{D}}{ }^{24}=+82.5(c$ $0.4, \mathrm{PhH}$ for $98 \%$ ee $)$; Lit. $^{9}[\alpha]_{\mathrm{D}}{ }^{24}=+27.4\left(c 1.10, \mathrm{CHCl}_{3}\right.$ for $58 \%$ ee) for $(S)-3 \mathbf{f} .{ }^{1} \mathrm{H}$ NMR (400 MHZ, $\left.\mathrm{CDCl}_{3}\right): 0.90(t, J=7,3 \mathrm{H}) ; 1.09-1.19(m, 1 \mathrm{H}) ; 1.37-1.52(m, 5 \mathrm{H})$; $3.34(s, 3 \mathrm{H}) ; 3.76(s, 3 \mathrm{H}) .{ }^{13} \mathrm{C}$ NMR (100 MHZ, $\left.\mathrm{CDCl}_{3}\right): 13.7(q) ; 21.5(t) ; 22.2(t)$; $28.2(d) ; 30.6(t) ; 33.9(s) ; 52.2(q) ; 52.4(q) ; 168.8(s) ; 171.4(s)$.

Enantiomer separation: chirasil- $\beta$-dex $(30 \mathrm{~m})$ column, oven temperature: $80{ }^{\circ} \mathrm{C}$ for 30 $\min$, then $1{ }^{\circ} \mathrm{C} / \min$ until $\left.180{ }^{\circ} \mathrm{C} . \mathrm{t}_{\mathrm{R}}[(S)-3 f)\right]=12.2 \mathrm{~min}, \mathrm{t}_{\mathrm{R}}[(R)-\mathbf{3 f}]=13.8 \mathrm{~min}$.

\section{Synthesis of substituted $\left[\operatorname{Rh}_{2}\{(S)-n t t l\}_{4}\right]$ catalysts.}

\subsection{Preparation of ligands}

\subsection{1. $N$-4-Chloro-1,8-naphthoyl-(S)-tert-leucine}

Purification by flash chromatography $\left(\mathrm{SiO}_{2}, \mathrm{EtOAc} / \mathrm{MeOH} 99: 1\right)$ gave 2.56g, (86\%) of $\left\{(S)-4-\mathrm{Cl}-\{\mathrm{nttl}\}\right.$ as colorless solid, m. p. 122. $[\alpha]_{\mathrm{D}}{ }^{24}=-53\left(c 0.1, \mathrm{CHCl}_{3}\right)$. IR (KBr): 3088w, 2956w, 2912w, 2862w, 1736m, 1703m, 1660s, 1585m, 1569m, 1366s, $1341 s, 1234 \mathrm{~s} .{ }^{1} \mathrm{H}$ NMR $\left(400 \mathrm{MHz}, \mathrm{CDCl}_{3}\right): 1.11(s, 9 \mathrm{H}) ; 5.48(s, 1 \mathrm{H}) ; 7.69(d, J=8$, $1 \mathrm{H}) ; 7.74(t, J=81 \mathrm{H}) ; 8.40-8.45, m, 1 \mathrm{H}) ; 8.44(d, J=8,1 \mathrm{H}) ; 8.55-8.65(m, 1 \mathrm{H}) .{ }^{13} \mathrm{C}$ NMR (100 MHz, $\left.\mathrm{CDCl}_{3}\right): 28.5(q) ; 36.1(s) ; 59.8(d) ; 124.2(s) ; 124.9(s) ; 128.9(s)$; $129.1(d) ; 130.7(d) ; 130.8(d) ; 131.7(s) ; 131.8(d) ; 132.3(d) ; 132.7(s) ; 163.8(s)$; $164.1(s) ; 173.6(s) . \mathrm{MS}: 345,347\left(\mathrm{M}^{+}, 0\right), 289\left(\mathrm{M}^{+}-\mathrm{C}_{4} \mathrm{H}_{8}, 26\right), 273(21), 272$ (10), 271 (62), 245 (10), 241 (12), 216 (29), 214 (10), 190 (11), 188 (34), 160 (15), 126 (12), 73 (100), 57 (40). HR MS: $289.0168\left(\mathrm{C}_{14} \mathrm{H}_{8} \mathrm{O}_{4} \mathrm{~N}^{35} \mathrm{Cl}^{+}\right.$; calc. 289.0142); 291.0139 $\left(\mathrm{C}_{14} \mathrm{H}_{8} \mathrm{O}_{4} \mathrm{~N}^{35} \mathrm{Cl}^{+}\right.$; calc. 291.0112).

\subsection{2. $N$-4-Bromo-1,8-naphthoyl-(S)-tert-leucine $\{(S)-4-B r-n t t l\} .{ }^{10}$}


Flash chromatography $\left(\mathrm{SiO}_{2}, \mathrm{EtOAc} / \mathrm{MeOH}\right.$ 99:1) afforded $\{(S)-4-\mathrm{Br}-\mathrm{nttl}\}$ (3.1g $92 \%)$ as white powder. M.p. 224. $[\alpha]_{\mathrm{D}}{ }^{24}=-40\left(c 0.1, \mathrm{CHCl}_{3}\right)$. IR (KBr): $2962 w$, $1704 s, 1665 s, 1586 m, 1571 m, 1366 s, 1341 s, 1238 m .{ }^{1} \mathrm{H}$ NMR (400 MHz, $\left.\mathrm{CDCl}_{3}\right)$ : $1.24(s, 9 \mathrm{H}) ; 5.62(s, 1 \mathrm{H}) ; 7.87-7.91(t, J=8,1 \mathrm{H}) ; 8.07(d, J=7.6,1 \mathrm{H}) ; 8.31-8.43(\mathrm{~m}$, $1 \mathrm{H}) ; 8.59(d, J=8.8,1 \mathrm{H}) ; 8.61-8.65(m, 1 \mathrm{H}) .{ }^{13} \mathrm{C} \mathrm{NMR}\left(100 \mathrm{MHz}, \mathrm{CDCl}_{3}\right): 28.5(q)$; $35.0(s) ; 59.8(d) ; 117.30(\mathrm{~s}) ; 118.2(\mathrm{~s}) ; 127.1(d) ; 127.6(s) ; 127.9(s) ; 129.6(s)$; $129.9(d) ; 130.2(d) ; 130.6(d) ; 131.0(d) ; 162.3(s) ; 162.8(s) ; 172.4(s)$. MS: 390 $\left(\mathrm{M}^{+}, 0\right), 347$ (13), 345 (13), $335\left(\mathrm{M}^{+}-\mathrm{C}_{4} \mathrm{H}_{8} 45\right), 333\left(\mathrm{M}^{+}-\mathrm{C}_{4} \mathrm{H}_{8}, 47\right), 330$ (11), 328 (10), 318 (16), 317 (100), 316 (17), 315 (100), 291 (16), 289 (20), 278 (24), 276 (24), 262 (39), 261 (10), 260 (52), 258 (14), 241 (30), 235 810), 234 (58), 233 (14), 232 (66), 206 (22), 204 (22), 197 (20), 153 (17), 152 (10), 151 (14), 126 (32), 125 (42), 99 (14), 75 (22), 74 (11), 57 (86), 55 (13). Anal. calc. for $\mathrm{C}_{18} \mathrm{H}_{16} \mathrm{BrNO}_{4}$ (390.24): C 55.40, H 4.13, N 3.59; found: C 55.77, H 4.25, N 3.19.

\subsection{3. $N$-4-Nitro-1,8-naphthoyl-(S)-tert-leucine $\left\{(S)-4-\mathrm{NO}_{2}\right.$-nttl $\}$.}

Flash chromatography $\left(\mathrm{SiO}_{2}, \mathrm{EtOAc} / \mathrm{MeOH} 99: 1\right)$ gave $\left\{(S)-4-\mathrm{NO}_{2}-\mathrm{nttl}\right\}(2.2 \mathrm{~g}, 72 \%)$ as colorless poweder. M.p. 136. $[\alpha]_{\mathrm{D}}{ }^{24}=-56\left(c 0.1, \mathrm{CHCl}_{3}\right)$. IR (KBr): $2956 w, 2902 w$, $2864 w, 1708 s, 1668 s, 1579 m, 1525 s, 1367 m, 1338 s, 1236 m .{ }^{1} \mathrm{H}$ NMR (400 MHz, $\left.\mathrm{CDCl}_{3}\right): 1.22(s, 9 \mathrm{H}) ; 5.60(s, 1 \mathrm{H}), 7.99(t, J=8,1 \mathrm{H}), 8.43(d, J=8,1 \mathrm{H}), 8.72-8.74(m$, $2 \mathrm{H}), 8.86(d, \mathrm{~J}=8,1 \mathrm{H}) .{ }^{13} \mathrm{C} \mathrm{NMR}\left(100 \mathrm{MHz}, \mathrm{CDCl}_{3}\right): 28.5(q) ; 36.1(d) ; 60.5(s)$; $122.5(s) ; 123.6(s) ; 123.9(d) ; 129.0(s) ; 129.6(d) ; 130.0(s) ; 133.2(d) ; 133.6(d)$; $149.7(s) ; 160.4(s) ; 160.7(s) ; 173.8(s) . \mathrm{MS}: 356\left(\mathrm{M}^{+}, 0\right) ; 300\left(\mathrm{M}^{+}-\mathrm{C}_{4} \mathrm{H}_{8}, 49\right), 283$ (17), 282 (100), 256 (19), 252 (23), 237 (13), 227 (34), 226 (13), 199 (31), 197 (15), 182 (10), 169 (11), 154 (12), 153 (22), 140 810), 126 (15), 125 (18), 57 (100), 55 (10). HR MS: $300.0375\left(\mathrm{C}_{14} \mathrm{H}_{8} \mathrm{O}_{6} \mathrm{~N}_{2}{ }^{+}\right.$; calc. 300.03824); $282.0281\left(\mathrm{C}_{14} \mathrm{H}_{6} \mathrm{O}_{5} \mathrm{~N}_{2}{ }^{+}\right.$; calc. 282.0277). Anal. calc. for $\mathrm{C}_{18} \mathrm{H}_{16} \mathrm{~N}_{2} \mathrm{O}_{6}$ (356.34): C 60.67, H 4.53, N 7.86; found: $\mathrm{C}$ $60.65, \mathrm{H} 4.50, \mathrm{~N}: 7.84$.

\subsection{Ligand exchange}

\subsubsection{Dirhodium(II) tetrakis[N-4-chloro-1,8-naphtholyl-(S)-tert-leucinate] $\left[\operatorname{Rh}_{2}\{(S)-4-C l-n t t l\}_{4}\right](4 b)$.}

Flash chromatography (alox basic, MeOH) afforded $\left[\mathrm{Rh}_{2}\{(S)-4-\mathrm{Cl}-\mathrm{nttl}\}_{4}\right](85 \%)$ as green solid. $[\alpha]_{\mathrm{D}}^{24}=+166\left(c 0.1, \mathrm{CHCl}_{3}\right)$. IR $(\mathrm{KBr}): 295 w, 1709 s, 1667 s, 1608 m$, 
$1591 m, 1575 m, 1399 m, 1366 m, 1344 s .{ }^{1} \mathrm{H}$ NMR (400 MHz, $\left.\mathrm{CDCl}_{3}\right): 1.31(s, 9 \mathrm{H}) ; 5.79$ $(s, 1 \mathrm{H}), 8.28-8.30(m ; 2 \mathrm{H}), 8.32(d, J=8,1 \mathrm{H}), 8.43(d, J=8,1 \mathrm{H}), 8.51-8.60 .(m, 1 \mathrm{H})$. ${ }^{13} \mathrm{C}$ NMR (100 MHz, $\left.\mathrm{CDCl}_{3}\right): 28.8(q) ; 29.4(q) ; 29.7(q), 36.2(s) ; 62.1(d) ; 121.8(s)$, $123.2(s) ; 123.3(s) ; 127.4(d) ; 127.8(d) ; 128.4(d) ; 128.9(s) ; 131.8(d) ; 132.2(d)$; $138.5(s) ; 162.4(s) ; 162.7 \quad(s) ; 186.8 \quad(s) . \quad \mathrm{MS} \quad(\mathrm{ESI}): 1605.0680 \quad\left(\mathrm{C}_{72} \mathrm{H}_{60}\right.$ ${ }^{35} \mathrm{Cl}_{4} \mathrm{~N}_{4} \mathrm{O}_{16} \mathrm{NaRh}_{2}{ }^{+}$; calc. 1605.0768

\subsubsection{Dirhodium (II) tetrakis[ $N$-4-bromo-1,8-naphthoyl-(S)-tert-leucinate] $\left[\operatorname{Rh}_{2}\{(S)-4-B r-n t t l\}_{4}\right](4 c)$.}

Flash chromatography (alox, basic, $\mathrm{MeOH}$ ) to afford $\left[\mathrm{Rh}_{2}\{(S)-4-\mathrm{Br}-\mathrm{nttl}\}_{4}\right]$ in $90 \%$ chemical yield. $[\alpha]_{\mathrm{D}}^{24}=+163\left(c 0.1, \mathrm{CHCl}_{3}\right)$. IR (KBr): 2950 (br), $1706(s), 1665(s)$, $1602(m), 1588(m), 1572(m), 1511(w), 1459(w), 1484(w), 1393(m), 1363(m)$, $1338(s), 1294(w), 1234(m), 1179(m), 1122(m), 1097(w), 905(m), 847(m), 773$ $(s), 713(m), 581(w), 526(m), 515(s), 499(s)$.

${ }^{1} \mathrm{H}$ NMR $\left.100 \mathrm{MHz}, \mathrm{CDCl}_{3}\right): 1.30(s, 9 \mathrm{H}) ; 5.79(s, 1 \mathrm{H}), 8.16(t, J=8,1 \mathrm{H}) ; 8.42(\mathrm{~d}, J$ $=8,1 \mathrm{H}), 8.57-8.59(m, 1 \mathrm{H}) ; 8.61(m, 1 \mathrm{H}), 8.83(d, J=8,1 \mathrm{H}) .{ }^{13} \mathrm{C}$ NMR $(100 \mathrm{MHz}$, $\left.\mathrm{CDCl}_{3}\right): 28.8(q) ; 29.4(q) ; 29.7(q), 36.1(s) ; 62.0(d) ; 122.2(s) ; 122.3(s) ; 128.8(d)$; $130.2(s) ; 130.4(s) ; 131.2(s) ; 131.9(d) ; 132.3(d) ; 132.8(d) ; 133.2(d) ; 162.5(s)$; $164.2(s) ; 187.0(s)$.

MS (ESI): $1780.8818 \quad\left(\mathrm{C}_{72} \mathrm{H}_{60}{ }^{79} \mathrm{Br}_{4} \mathrm{~N}_{4} \mathrm{O}_{16} \mathrm{Rh}_{2} \mathrm{Na}^{+} ;\right.$calc. 1780.8740); 902.9670 $\left(\mathrm{C}_{72} \mathrm{H}_{60}{ }^{79} \mathrm{Br}_{4} \mathrm{~N}_{4} \mathrm{O}_{16} \mathrm{Rh}_{2} \mathrm{Na}_{2}{ }^{++}\right.$; calc. 901.9316).

\subsubsection{Dirhodium(II) tetrakis[ $N$-4-nitro-1,8-naphthoyl-(S)-tert-leucinate] $\left[\operatorname{Rh}_{2}\left\{(S)-4-\mathrm{NO}_{2}-\text {-nttl }\right\}_{4}\right](4 d)$.}

Flash chromatography (alox, basic, $\mathrm{MeOH}$ ) afforded $\left[\mathrm{Rh}_{2}\left\{(S)-4-\mathrm{NO}_{2}-\mathrm{nttl}\right\}_{4}\right]$ in $90 \%$ chemical yield. $[\alpha]_{\mathrm{D}}{ }^{24}=+122\left(c 0.1, \mathrm{CHCl}_{3}\right)$. IR (KBr): $2927(b r), 1712(s), 1667(s)$, $1609(m), 1528(s), 1464(w), 1397(s), 1337(s), 1300(w), 1238(s), 1184(m), 1128$ $(w), 910(m), 786(s), 758(\mathrm{~s}), 712(m), 510(s) 481(s) .{ }^{1} \mathrm{H}$ NMR $\left.400 \mathrm{MHz}, \mathrm{CDCl}_{3}\right)$ : $1.28(s, 9 \mathrm{H}) ; 5.78(s, 1 \mathrm{H}) ; 7.89-7.90(m, 1 \mathrm{H}) ; 8.65(d J=4,1 \mathrm{H}), 8.66-8.74(m, 1 \mathrm{H})$, $8.85(t J=8,1 \mathrm{H}), 8.89(m, 1 \mathrm{H}) .{ }^{13} \mathrm{C} \mathrm{NMR}\left(100 \mathrm{MHz}, \mathrm{CDCl}_{3}\right): 28.7(q) ; 29.3(q) ; 29.5$ $(q), 36.2(s) ; 62.1(d) ; 122.9(s), 123.4(s) ; 123.4(d) ; 127.0(s) ; 128.8(d) ; 129.1(s)$; $129.8(d) ; 130.9(d) ; 133.4(d) ; 149.3(s) ; 162.3(s) ; 163.1(s) ; 186.9(s)$.

MS (ESI): $1649.1872\left(\mathrm{C}_{72} \mathrm{H}_{60} \mathrm{~N}_{8} \mathrm{O}_{24} \mathrm{Rh}_{2} \mathrm{Na}^{+}\right.$; calc. 1649.1736). 


\section{Enantiomer separation}

Enantiomer separation of $\mathbf{3 b}$ with ligand $\mathbf{4 c}(65 \%$ ee)<smiles>COC(=O)C1(C(C)=O)CC1c1ccc(Cl)cc1</smiles><smiles>CC(C)(C)C(C(=O)O)N1C(=O)c2cccc3c(Br)ccc(c23)C1=O</smiles>

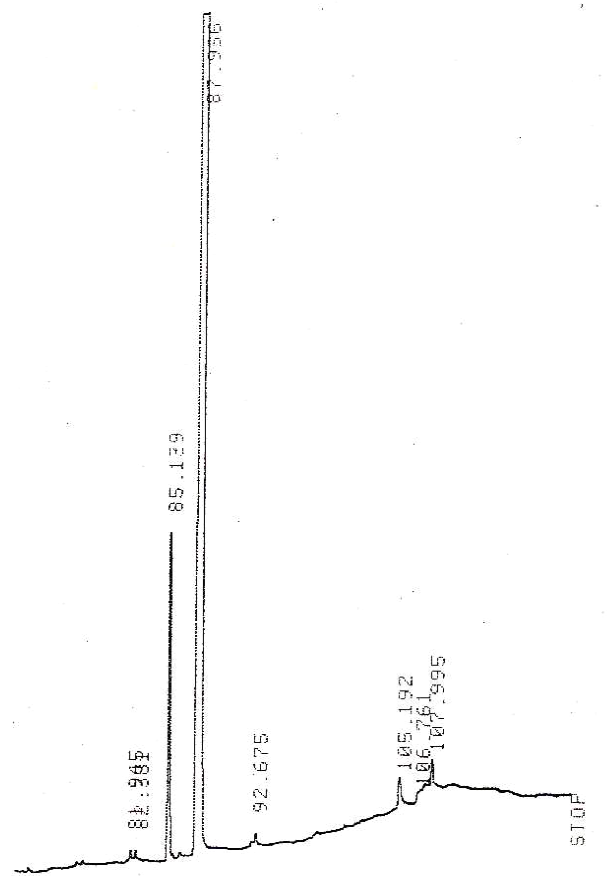

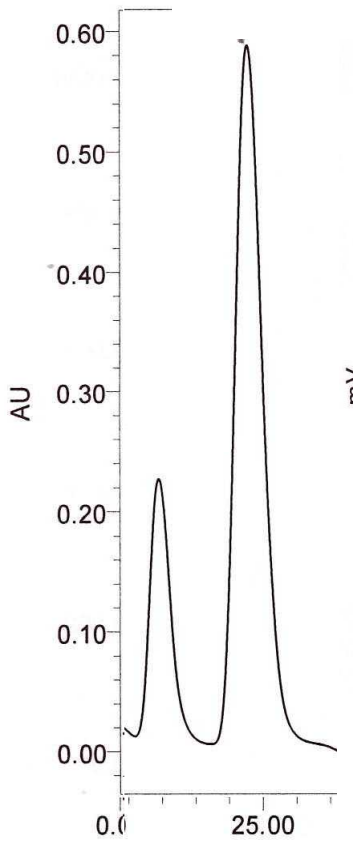

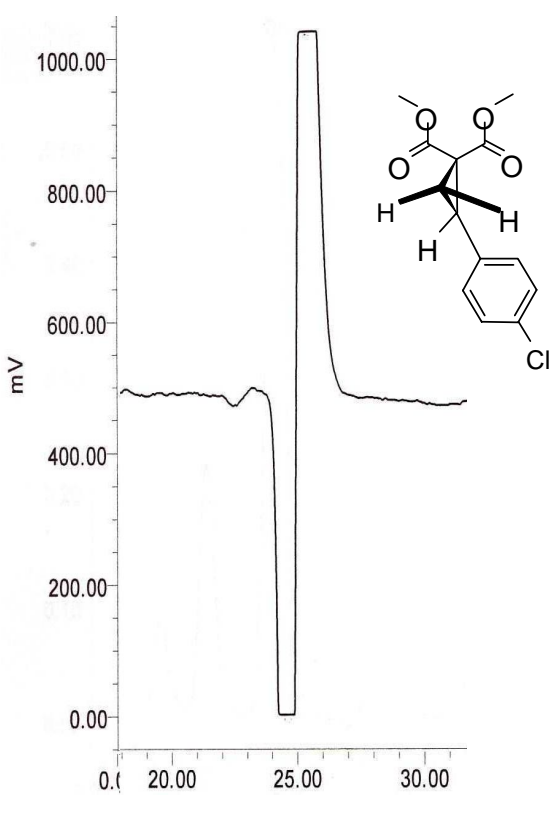

Enantiomer separation of $\mathbf{3 c}$ with ligand $\mathbf{4 c}(\mathbf{8 7 \%} \mathrm{ee})$
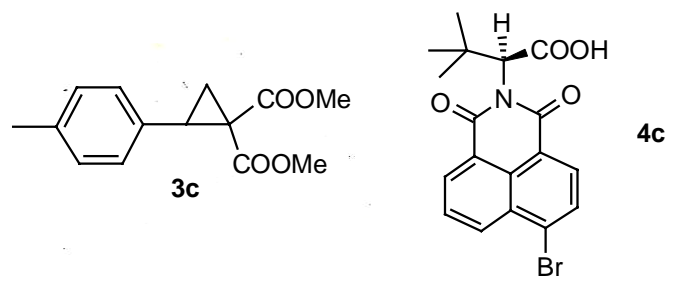

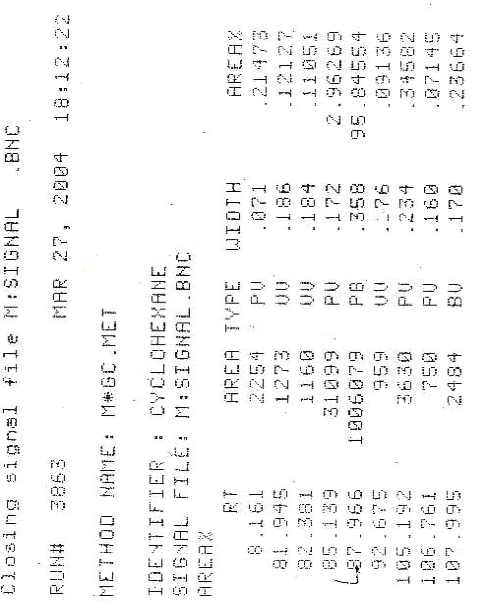


Enantiomer separation of $\mathbf{3 f}$ with ligand $\mathbf{4 c} \quad 98 \%$ ee)

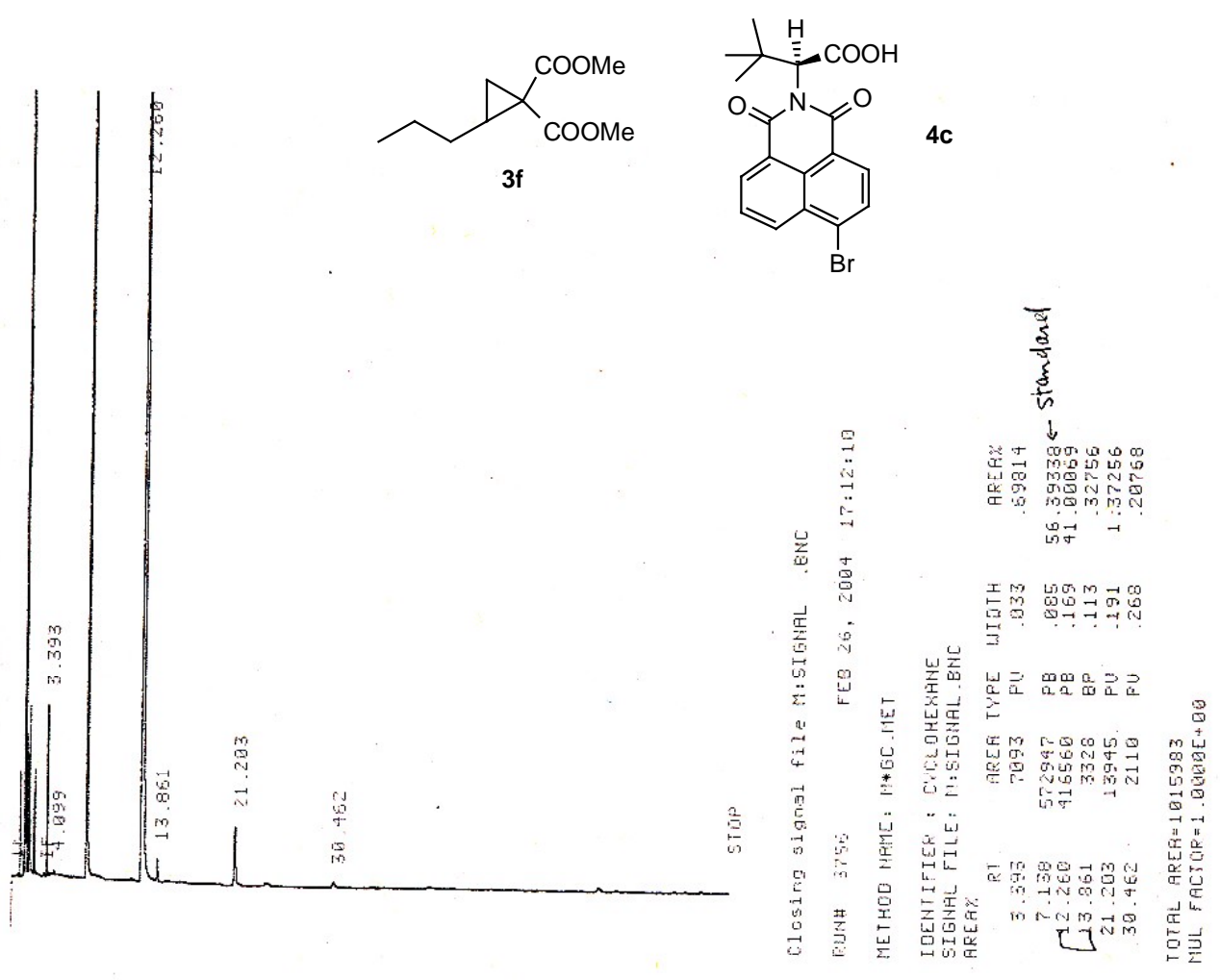

Enantiomer separation of $\mathbf{6 a}$ with ligand $\mathbf{4 c}(92 \% \mathrm{ee})$

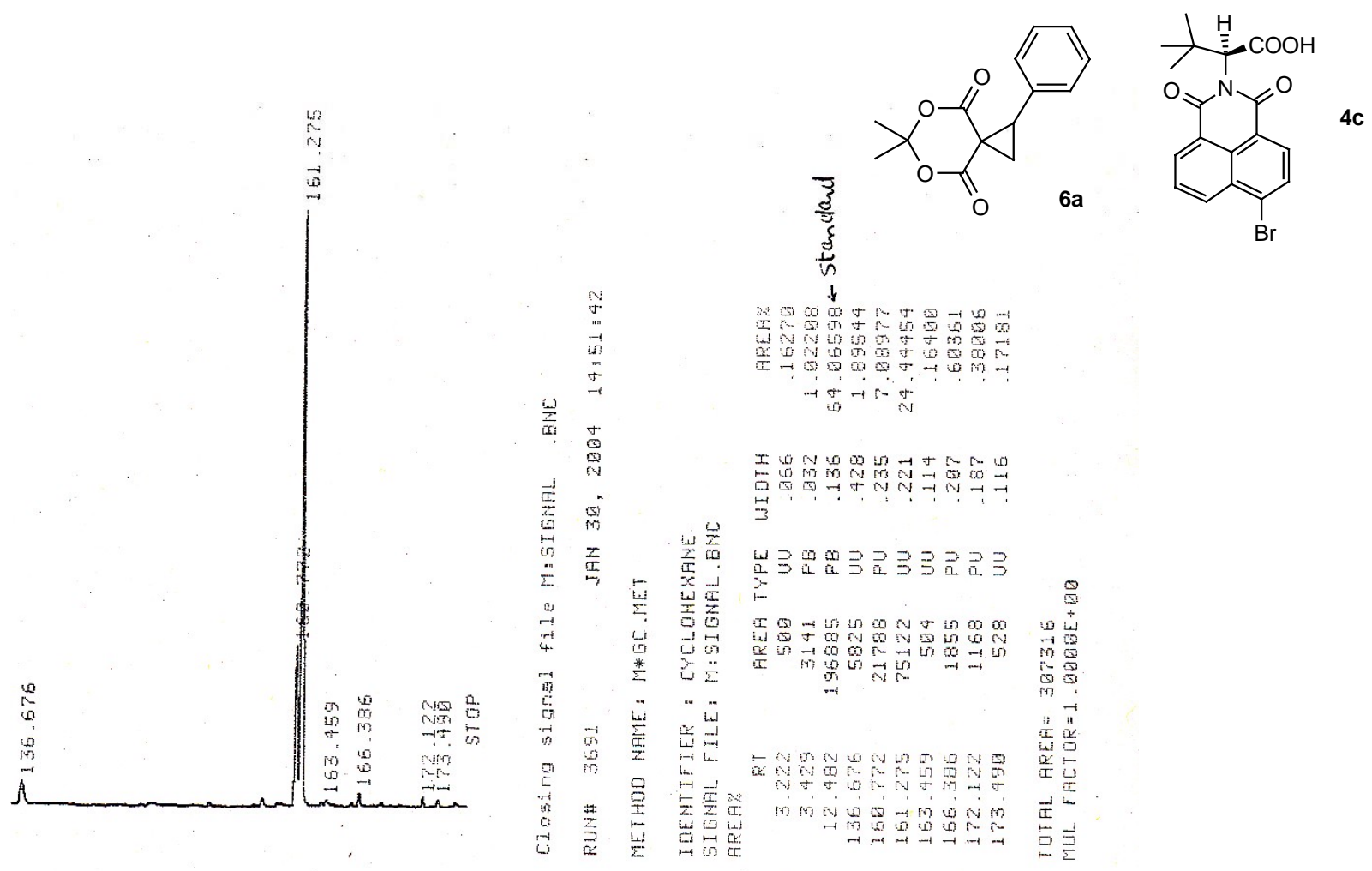



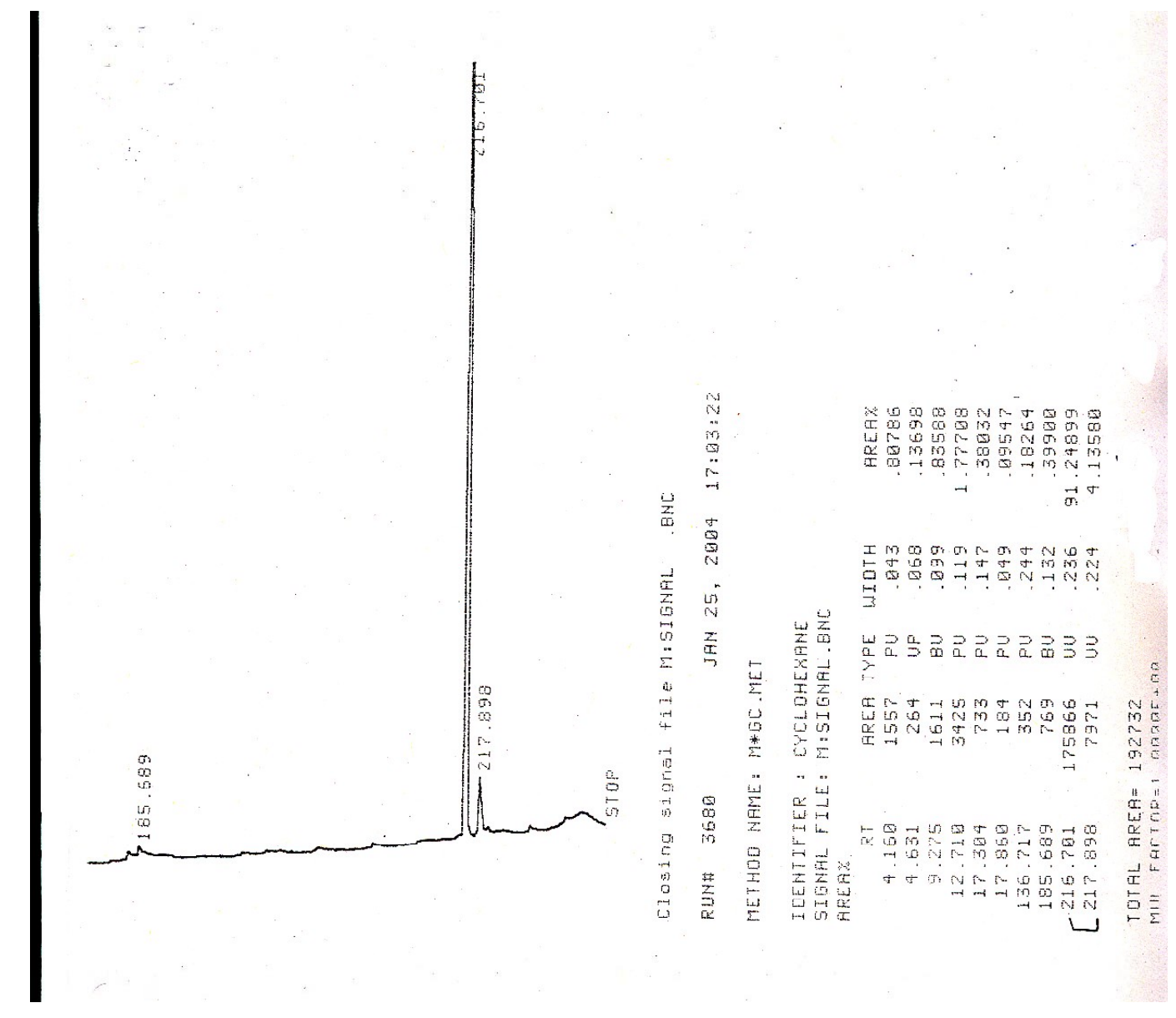


\section{References}

${ }^{1}$ Dauban, P.; Sanière, L.; Tarrade, A.; Dodd, R. H. J. Am. Chem. Soc. 2001, 123, 7707-7708.

${ }^{2}$ Dietrich, A.; Maas, B.; Messer, W.; Bruche, G.; Volker, K.; Kaunzinger, A.;

Mosandl, A. J. High Resolut. Chromatogr. 1992, 15, 590-593.

${ }^{3}$ Ghanem, A.; Ginatta, C.; Jiang, Z.; Schurig, V. Chromatographia 2003, 57

(Suppl.), S/275-S/281.

${ }^{4}$ Müller, P.; Fernandez, D. Helv. Chim. Acta 1995, 78, 947-958.

${ }^{5}$ Corey, E. J.; Grant, T. G. Tetrahedron Lett. 1994, 35, 5373-5379.

${ }^{6}$ (a) Davies, H. M. L.; Panaro, S. A. Tetrahedron 2000, 56, 4871-4880. (b) Nymann, K.; Svendsen, J. S. Acta Chem. Scand. 1998, 52, 338-349.

${ }^{7}$ Sato, M; Hisamichi, H; Kaneko, C.; Suzaki, N.; Furuya, T.; Inukai, N. Tetrahedron Lett. 1989, 30, 5281-5284.

${ }^{8}$ Doyle, M. P.; Hu, W. ARKIVOC 2003, 7, 15-22; Doyle, M. P.; Davies, S. B.; Hu, W. Org. Lett. 2000, 2, 1145-1147.

9 Müller, P.; Allenbach, Y.; Robert, E. Tetrahedron: Asymmetry 2003, 14, 779-785.

${ }^{10}$ Hoshino, Y.; Yamamoto, H. J. Am. Chem. Soc. 2000, 122, 10452-10453. 